\title{
The Reproductive Outcomes for the Infertile Patients With Recurrent Implantation Failures May Be Improved by Endometrial Receptivity Array Test
}

\author{
Takeharu Ota ${ }^{\mathrm{a}}$, Miyako Funabiki ${ }^{\mathrm{a}}$, Yoshihiro Tada ${ }^{\mathrm{a}}$, Masako Karita ${ }^{\mathrm{a}}$, Terumi Hayashi ${ }^{\mathrm{a}}$, \\ Kazunori Maeda ${ }^{a}$, Takashi Matsubara ${ }^{a}$, Yuri Iwaki ${ }^{a}$, Naomi Sugiyama ${ }^{a}$, \\ Takashi Henmi ${ }^{\text {a }}$ Noriko Watanabe ${ }^{a}$, Yoshitaka Nakamura ${ }^{\text {a }}$ \\ Sagiri Taguchi ${ }^{a, b}$
}

\begin{abstract}
We report a successful first birth case from a patient with refractory recurrent implantation failure (RIF) by using endometrial receptivity array (ERA) test in Japan. The reproductive outcomes for the infertile patients with recurrent implantation failures may be improved by ERA test.
\end{abstract}

Keywords: Recurrent implantation failures; Endometrial receptivity array test; Reproductive outcomes

\section{Introduction}

Endometrial sensitivity assay has been in practice for some time. However, conventional endometrial sensitivity assay is not an innovative technology in infertility. In order to exceed the limit of conventional endometrial sensitivity assay, as a new tool for the treatment of the infertile patients with recurrent implantation failures, the endometrial receptivity array (ERA) test is developed [1]. The ERA test is used in order to diagnose the receptivity of the endometrium by a specific transcriptomic signature [1]. Furthermore, the ERA examines 236 genes expressed at different stages of the endometrial cycle and thereby defines the endometrium as either receptive or non-receptive. As a result, it is shown that the ERA test can identify window of implantation (WOI) and adjust the timing of embryo transfer [2].

However, the improvement of the reproductive outcomes for the infertile patients with recurrent implantation failures by

Manuscript submitted March 10, 2019, accepted April 3, 2019

aIVF center, Oak Clinic, Osaka, Japan

bCorresponding Author: Sagiri Taguchi, IVF center, Oak Clinic, 2-7-9 Tamade-Nishi, Nishinari-ku, Osaka, 557-0045, Japan.

Email: taguchi_s@oakclinic-group.com

doi: https://doi.org/10.14740/jmc3282
ERA test is controversial [3-4]. Therefore, innovativeness of ERA is not clear.

\section{Case Report}

A 34-year-old woman with infertility visited at our clinic in 2014. Semen examination, hysterosalpingography, hormone examination and anti-sperm antibody were carried out for the patient at our clinic. However, abnormalities were not observed. Therefore, as unexplained infertility, we conducted Timing Method (four times) and AIH (three times) for the patient, but it did not lead to pregnancy and shifted to in vitro fertilization. Specifically, the Timing Method is a treatment that diagnoses ovulation day and adjusts the timing of sexual intercourse. Furthermore, the AIH means artificial insemination by husband.

After that, until August 2016, thawed frozen embryos with good morphological blastocysts were transplanted for the patient under hormone replacement therapy cycle (11 times). However, the patient could not experience live birth. During that period the patient experienced chemical pregnancy (four times), miscarriage (one time) and negative pregnancy reaction (six times). Specifically, negative pregnancy reactions mean that chemical pregnancy and/or clinical pregnancy is not observed. From these clinical processes, we diagnosed the patient as recurrent implantation failure (RIF). Furthermore, although we performed coagulation test, anti-phospholipid antibody test, blood test of thyroid function and hysteroscopy as cause search of RIF, abnormalities were not observed for the patient.

Therefore, we tried to identify window of implantation and adjust the timing of thawed frozen embryo transfer by ERA test with the patient's informed consent. As a result, the patient could experience live birth by a cesarean section (38 weeks, 3,272 g, female) in 2017. The baby survives without birth defects.

Furthermore, we confirm that the work in this study was conducted in accordance with the Code of Ethics of the World Medical Association (Declaration of Helsinki). Moreover, all of the experiments were approved by the 


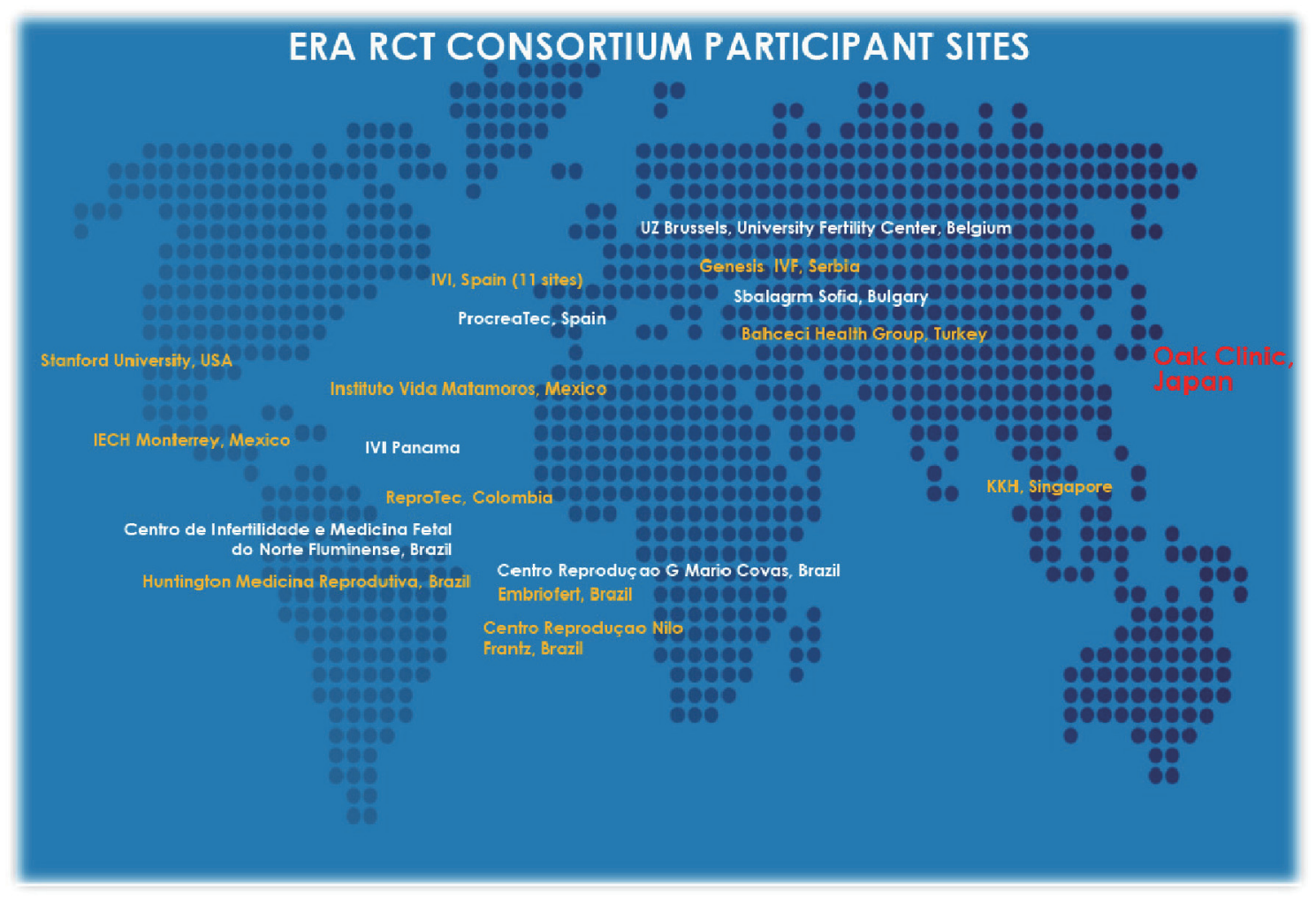

Figure 1. ERA RCT consortium participant sites.

institutional review board of Oak Clinic in Japan. In addition, our report was written in accordance with Case Report guidelines (CARE: https://www.care-statement.org/carechecklist.html).

\section{Discussion}

In our report, ERA test was conducted for the patient with refractory RIF, and the pregnancy condition continued smoothly, and it was possible to get a live birth. This is the first successful birth case from a patient with refractory RIF by using ERA test in Japan, although we have introduced ERA test from November, 2014 for the first time in Japan.

The results of ERA test are checked at the genetic levels [2]. When we can find that the patient's WOI is shifted by the ERA test, it is possible to identify the WOI period of the patient and adjust the optimal timing of embryo transfer [2]. Therefore, as embryo transfer can be performed at an appropriate timing by clarifying the WOI period at a genetic level, the improvement of the reproductive outcomes would be expected. From the above, we think that the ERA test may realize precision medicine [5] in the field of reproductive medicine.

However, from the viewpoint of evidence-based medicine, in order to truly clarify the effectiveness of ERA test, it is necessary to conduct randomized controlled trials and to verify the results. Therefore, we participate in an international randomized controlled trial (prospective, randomized, multi- center, international, open label, controlled trial: UMIN ID, UMIN000022044; Fig. 1) as the only Japanese institute.

\section{Acknowledgments}

We are grateful to all members at the Oak Clinic in Japan.

\section{Financial Disclosure}

The present study is self-funded.

\section{Conflict of Interest}

We have no conflict of interest.

\section{Informed Consent}

The patient's informed consent was obtained for the preparation of this case report.

\section{Author Contributions}

All authors contributed equally to this work. 


\section{References}

1. Diaz-Gimeno P, Horcajadas JA, Martinez-Conejero JA, Esteban FJ, Alama P, Pellicer A, Simon C. A genomic diagnostic tool for human endometrial receptivity based on the transcriptomic signature. Fertil Steril. 2011;95(1):5060, e51-15.

2. Oviedo Choy J, Godoy Morales H, Cordova Castillo M, Merchand Alvarez P, Lozano M. Endometrial receptivity genetic expression and histological analysis in women with repeated implantation failure. In: Gynecological Endocrinology; Vol 32. Oxon, England: Taylor \& Francis Ltd, 4 Park
Square, Milton Park, Abingdon OX14 4RN, 2016;57-58.

3. Hashimoto T, Koizumi M, Doshida M, Toya M, Sagara E, Oka N, Nakajo Y, et al. Efficacy of the endometrial receptivity array for repeated implantation failure in Japan: A retrospective, two-centers study. Reprod Med Biol. 2017;16(3):290-296.

4. Bassil R, Casper R, Samara N, Hsieh TB, Barzilay E, Orvieto $R$, Haas J. Does the endometrial receptivity array really provide personalized embryo transfer? J Assist Reprod Genet. 2018;35(7):1301-1305.

5. Collins FS, Varmus H. A new initiative on precision medicine. N Engl J Med. 2015;372(9):793-795. 